\title{
Changes in QRS and T-wave Loops Subsequent to an Increase in Left Ventricle Globularity as in Intrauterine Growth Restriction: a Simulation Study
}

\author{
Freddy L Bueno-Palomeque ${ }^{1,2,3}$, Konstantinos A Mountris ${ }^{1,2}$, Ana Mincholé ${ }^{4}$, Nuria Ortigosa ${ }^{5}$, \\ Raquel Bailón ${ }^{1,2}$, Esther Pueyo ${ }^{1,2}$, Pablo Laguna ${ }^{1,2}$ \\ ${ }^{1}$ BSICoS Group, I3A, IIS Aragón, Universidad de Zaragoza, Zaragoza, Spain \\ ${ }^{2}$ CIBER en Bioingeniería, Biomateriales y Nanomedicina (CIBER-BBN), Zaragoza, Spain \\ ${ }^{3}$ GIIB-UPS, Universidad Politécnica Salesiana, Cuenca, Ecuador \\ ${ }^{4}$ M2BE Group, Mechanical Engineering Department, Universidad de Zaragoza, Zaragoza, Spain \\ ${ }^{5}$ I.U. Matemática Pura y Aplicada, Universitat Politècnica de València, Spain
}

\begin{abstract}
Cardiovascular remodeling induced by intrauterine growth restriction manifests in adulthood by more globular ventricles, as evidenced by in vivo measurements. The angle between the dominant vectors of the QRS and T-wave loops has been reported to be significantly altered as a result of the induced remodeling. To investigate whether the more globular ventricular shape was a major factor contributing to such alteration, we performed electrophysiological simulations in a human biventricular model for control and in a model obtained by deforming the control one to represent a more spherical left ventricle (SLV). Transmural ventricular heterogeneities and a Purkinje network were included. 12-lead ECGs were calculated, from which spatial QRS and T-wave angles were computed. The angle between the T-wave and the XZ-plane was found to increase in the SLV model, showing a variation similar to that reported in in vivo studies. However, the angle between the dominant vectors of the QRS and T-wave loops projected onto the XY-plane was lower for control, contrary to clinical observations in IUGR adults. Other clinical results could not be reproduced in our simulations either. Our findings suggest that a more globular left ventricular shape leads to changes in the angles of QRS and T-wave loops, but further research is needed to fully understand these changes and the underlying mechanisms.
\end{abstract}

\section{Introduction}

Intrauterine growth restriction (IUGR) and low birth weight are factors predisposing to increased risk of heart disease in adulthood [1]. IUGR is associated with cardiovascular remodeling, which manifests by more globular ventricles when assessed by the sphericity index, defined as the ratio between the base-to-apex length and the basal diameter. Specifically, individuals who had IUGR present an increase in basal diameter, a reduction in baseto-apex length and, consequently, a more globular ventricle [2]. Previous studies on electrocardiographic (ECG) signals from adult individuals who suffered IUGR have shown that the angle between QRS and T-wave dominant vectors is significantly different than in control individuals, possibly reflecting the cardiovascular remodeling subsequent to IUGR [3]. Importantly, such changes have been suggested to be associated with higher cardiovascular mortality [1].

The impact of IUGR-induced remodeling in the heart over the course of time has been investigated in the literature. In children and preadolescents, studies including echocardiography, blood pressure and/or ECG measurements have evidenced that changes in the anatomy or function of the heart persist at six months of age and after more than 10 years later $[2,4]$. Research on adult individuals has corroborated some of the results observed in children and preadolescents. Over vectorcardiogram signals generated from standard 12-lead ECGs, statistically significant differences between individuals who suffered IUGR and controls were found in the angle between the dominant vector of the QRS-complex and the dominant vector of T-wave projected on the frontal plane [3]. These variations were postulated as possible markers of IUGR-induced cardiovascular risk. Currently, little is known about the morphophysiological factors that cause this angular variation in individuals who had IUGR and the potential mechanisms underlying its relationship with cardiovascular risk in adulthood. In this study, we conducted an electrophysiological modeling and simulation study using human biventricular geometries for control and for spherical left ventricle (SLV). From the simulated electrical activity in each of the two geometries, ECG signals were calculated and the angles between the dominant vectors of the QRS-complex 
and T-wave loops were evaluated. The extent to which the simulated change in LV shape could explain differences in the quantified angles as those reported in the literature for adults who suffered IUGR were determined.

\section{Materials and methods}

A control biventricular geometry was obtained from image data of a patient with no history of cardiovascular disease. By applying loads onto this control geometry, another SLV geometry was derived as in in vivo studies.

The three-dimensional biventricular models included transmural heterogeneities, corresponding to endocardial, midmyocardial and epicardial tissues in proportions of $50 \%, 20 \%$ and $30 \%$ of the wall thickness, respectively. Human ventricular cell electrophysiology was represented by the O'Hara-Rudy action potential (AP) model.

Muscle fiber direction was assigned to each node of the tetrahedral mesh using a rule-based method [5]. The value of the longitudinal diffusion coefficient was 0.0025 $\mathrm{cm}^{2} / \mathrm{ms}$ and the transversal-to-longitudinal conductivity ratio was set to 0.25 . Purkinje fibers for electrical stimulation were implemented in the model using a method based on fractal projection [6]. The complete His-Purkinje network was modeled by additionally including the bundle branch, which was divided into three different parts, as in [7], following ex vivo and in vivo experimental reports (Figure 1). In the right ventricle, one part reaches the anteroapical region. In the left ventricle, one part reaches the posterobasal location and the other one, the apex. The diffusion coefficient in the conduction system was set to $0.0068 \mathrm{~cm}^{2} / \mathrm{ms}$. In the Purkinje-myocardial junctions (PMJ), the diffusion coefficient was $0.0013 \mathrm{~cm}^{2} / \mathrm{ms}$ and we incorporated a transition stage between each PMJ and the endocardium [8].
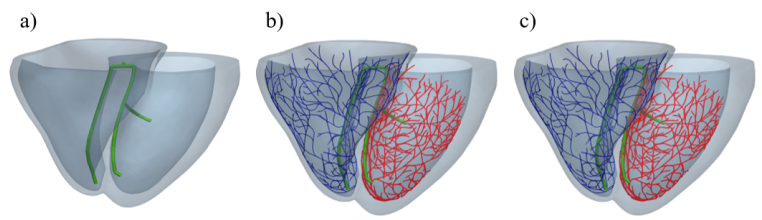

Figure 1. Biventricular model. a) Bundle brunch, b) Purkinje network on the control model, c) Purkinje network on the SLV model.

Simulations were performed using the software ELECTRA implementing the Finite Element Method, used in this study to solve the monodomain model of electrical propagation in cardiac tissue [9]. APs were simulated while pacing at a cycle length of $1000 \mathrm{~ms}$. The stimulus current applied onto the His Bundle had an amplitude of $200 \mathrm{~mA}$ and a duration of $0.5 \mathrm{~ms}$.

12-lead ECGs were simulated by placing virtual elec- trodes in the standard ECG lead positions on the torso and computing the extracellular potentials [10]. From the simulated ECGs, the angles between the dominant QRS and T-wave vectors were computed using the method described in [3] and compared with the results reported there for adult individuals who had IUGR and controls.

\section{Results}

The control model had a sphericity index of 1.54 (baseto-apex length $=70.73 \mathrm{~mm}$, basal diameter $=45.83 \mathrm{~mm}$ ), whereas the SLV model 1.32 (base-to-apex length $=63.66$ $\mathrm{mm}$, basal diameter $=48.17 \mathrm{~mm}$ ), which is in line with the mean IUGR-related sphericity change reported in [2].

The transformation from 12-lead ECGs to orthogonal leads X, Y and Z was based on the inverse Dower's method [11] and are presented in figure 2 for the control and SLV geometries.
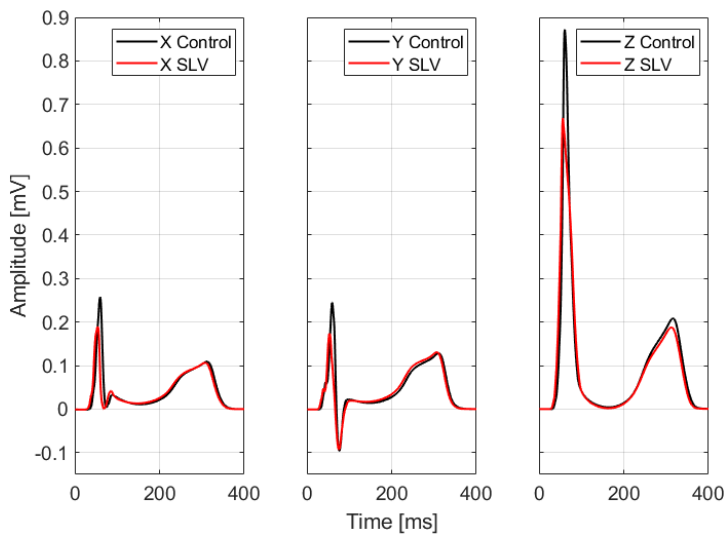

Figure 2. Orthogonal leads X, Y, and Z derived from 12lead pseudo-ECG.

The angles between the dominant vector of the depolarization and repolarization loops, shown in Figure 3, as well as the projections onto different planes are presented in Table 1 for control and SLV models. These results are compared with those obtained in vivo in control adults with term birth and normal weight for gestational age and in adults with IUGR at birth [3]. As can be seen from the table, the angle between the QRS and T-wave loops $\left(\theta_{R T}\right)$ both projected onto XY-plane (frontal plane), was lower for control, which disagrees with results presented in [3].

The angle between QRS loop $\left(\phi_{R}\right)$ and the XZ (transverse plane) and YZ (sagittal plane) planes, decreased in the SLV model, whereas it increased when computed for the XY-plane. The angle of the T-wave $\left(\phi_{T}\right)$ projected on the $\mathrm{XZ}$ and $\mathrm{YZ}$ planes, showed an increase in the SLV model, while it showed a reduction for the XY-plane. The difference between the dominant vectors of the QRS and T-wave loops $\left(\phi_{R}-\phi_{T}\right)$ projected on the three planes, all 
Table 1. Mean \pm standard deviation of loop angles: $*$ p-value $<0.05$, $* *$ p-value $<0.01$ indicate significant differences between control and IUGR groups in [3].

\begin{tabular}{ccccc}
\hline \hline \multicolumn{5}{c}{ Adults } \\
\hline Angle & Control & IUGR & Control Simulated & SLV Simulated \\
\hline (degrees) & $\mathrm{n}=54$ & $\mathrm{n}=33$ & $\mathrm{n}=1$ & $\mathrm{n}=1$ \\
\hline$\theta_{R T-X Y}$ & $13.49 \pm 13.65$ & $9.26 \pm 8.47 * *$ & 14.77 & 23.83 \\
$\phi_{R-X Z}$ & $32.85 \pm 9.08$ & $35.68 \pm 6.12$ & 9.21 & 5.60 \\
$\phi_{R-X Y}$ & $29.47 \pm 13.02$ & $22.07 \pm 11.80 * *$ & 73.52 & 77.40 \\
$\phi_{R-Y Z}$ & $40.72 \pm 12.05$ & $44.17 \pm 9.81$ & 13.53 & 11.24 \\
$\phi_{T-X Z}$ & $29.39 \pm 10.61$ & $33.15 \pm 10.67 *$ & 30.05 & 33.01 \\
$\phi_{T-X Y}$ & $14.42 \pm 8.68$ & $14.48 \pm 9.41$ & 48.54 & 45.01 \\
$\phi_{T-Y Z}$ & $54.83 \pm 9.52$ & $51.52 \pm 12.13$ & 25.65 & 26.76 \\
$\phi_{R-X Z}-\phi_{T-X Z}$ & $3.45 \pm 9.61$ & $2.54 \pm 8.43$ & -20.84 & -27.41 \\
$\phi_{R-X Y}-\phi_{T-X Y}$ & $15.05 \pm 14.14$ & $7.59 \pm 14.58 * *$ & 24.97 & 32.38 \\
$\phi_{R-Y Z}-\phi_{T-Y Z}$ & $-14.11 \pm 13.99$ & $-7.35 \pm 13.96 * *$ & -12.11 & -15.51 \\
\hline \hline
\end{tabular}

increased for the SLV model. Of all these results, only the ones referred to $\phi_{T-X Z}$ agree with those reported in [3] for IUGR individuals vs controls.

\section{Discussion}

Electrophysiological simulations were conducted in a control biventricular model and a more globular model obtained from it to simulate the change in sphericity induced by IUGR. Characterization of the angle between the dominant vectors of depolarization and repolarization projected onto different planes showed differences between the control and SLV models, but these were in most cases not concordant with the results presented in [3] for adults who had IUGR vs controls. Specifically, out of the five angles that were shown to be significantly different for control and IUGR groups in [3], we could only reproduce similar results for one of them.

In our study, the angle between the T-wave loop and the XZ-plane increased in the SLV model, showing a variation similar to that reported in [3] for IUGR individuals vs controls. On the other hand, the angle between the QRS and T-wave loops on the XY-plane $\left(\theta_{R T-X Y}\right)$ increased by 9 degrees in the SLV model with respect to control, whereas in [3] it was significantly reduced in the IUGR group. Similarly, we observed an increase in the angle between the QRS loop and the XY-plane $\left(\phi_{R-X Y}\right)$ for the SLV model, while results in the literature indicated that IUGR individuals had a reduction in that angle. The other two angles showing significant differences in [3] were those between the dominant vectors of QRS and T-wave loops in the XY and YZ planes. The change in IUGR vs control was opposite to the one we found for SLV vs control.

One potential reason underlying the discrepancies between our results and those in the literature might lie on the fact that the $\mathrm{Z}$ lead presents an amplitude notably higher than $\mathrm{X}$ and $\mathrm{Y}$ leads. This may have an important impact on the calculation of the dominant vectors of the QRS and T-wave loops and, thus, on their angles and should be investigated in future studies.

Several limitations should be acknowledged. The SLV geometry was built by applying loads onto the control geometry, but more elaborated methods to artificially built spherical LV geometries as well as the use of geometries from patients with and without IUGR will be explored in future studies. Also, a fixed proportion of transmural heterogeneities was simulated, while it is well known that variations in such heterogeneities markedly influence ECG repolarization [12]. A fractal-based structure of the Purkinje network was incorporated into the biventricular models to make electrical propagation more realistic. However, its impact on ventricular activation and repolarization should be further analyzed.

\section{Conclusions}

Electrophysiological simulations in biventricular models representative of control and of globular shape associated with IUGR-induced remodeling reproduce only partially the changes in the angles of the QRS-complex and T-wave loops reported in patients. Further research is required to shed light on the factors contributing to IUGRinduced changes in ECG depolarization and repolarization and the involved mechanisms.

\section{Acknowledgments}

I would like to acknowledge support by Fundación Carolina, Universidad de Zaragoza and Universidad Politécnica Salesiana through its doctoral scholar- 

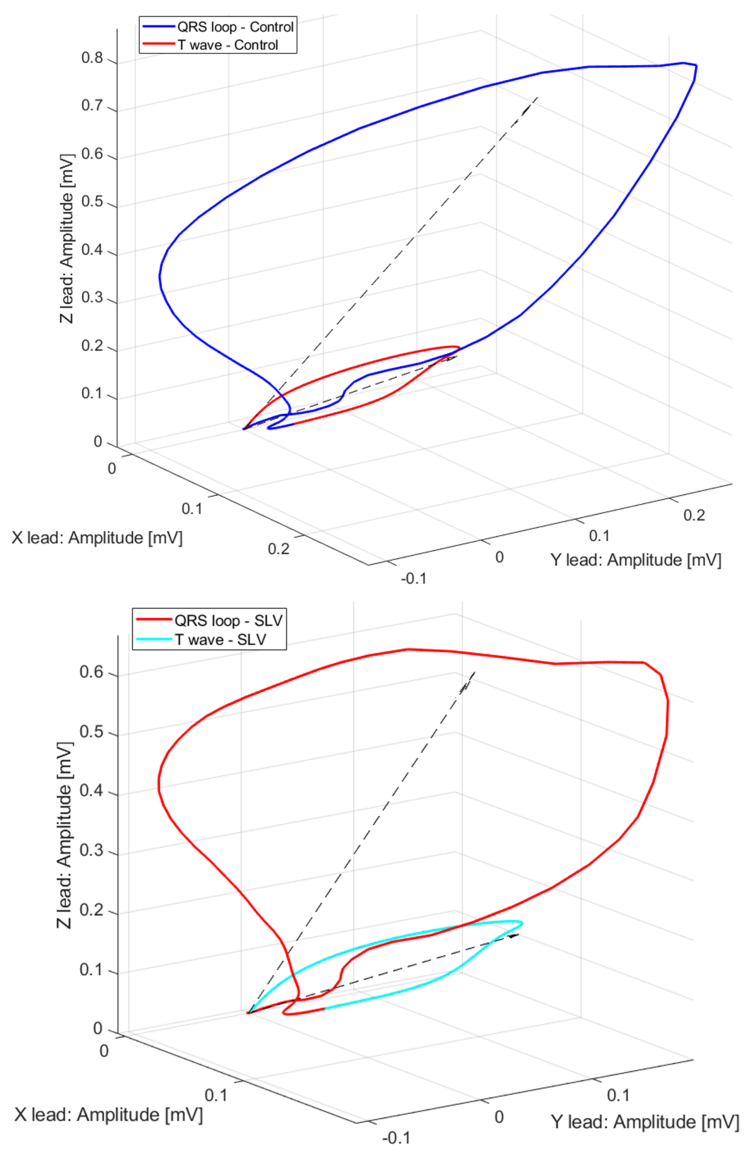

Figure 3. Vectorcardiogram 3D of depolarization loop (QRS) and repolarization (T) with its dominant vectors.

ship. This work was supported by projects ERC-StG 638284 (ERC), PID2019-105674RB-I00 and PID2019104881RB-I00 (MICINN) and LMP124-18 and reference group T39-20R (Aragón Government cofunded by FEDER 2014-2020 "Building Europe from Aragon"). Computations were performed using ICTS NANBIOSIS (HPC unit at U. Zaragoza). N. Ortigosa acknowledges support from Generalitat Valenciana under grant Prometeo/2017/102 and Spanish MINECO under grant MTM2016-76647-P.

\section{References}

[1] Ueda P, Cnattingius S, Stephansson O, Ingelsson E, Ludvigsson JF, Bonamy AKE. Cerebrovascular and Ischemic heart disease in young adults born preterm: A populationbased Swedish cohort study. European Journal of Epidemiology apr 2014;29(4):253-260. ISSN 15737284.

[2] Sarvari SI, Rodriguez-Lopez M, Nuñez-Garcia M, Sitges M, Sepulveda-Martinez A, Camara O, Butakoff C, Gratacos E, Bijnens B, Crispi F. Persistence of cardiac remodeling in preadolescents with fetal growth restriction. Circulation Cardiovascular Imaging jan 2017;10(1). ISSN 1941-9651.

[3] Ortigosa N, Rodriguez-Lopez M, Bailon R, SepulvedaMartinez A, Gratacos E, Crispi F, Laguna P. Intrauterine growth restriction induced ECG morphological differences measured in adulthood. Computing in Cardiology 2018; 2018-Septe:3-6. ISSN 2325887X.

[4] Cruz-Lemini M, Crispi F, Valenzuela-Alcaraz B, Figueras F, Sitges M, Bijnens B, Gratacós E. Fetal cardiovascular remodeling persists at 6 months in infants with intrauterine growth restriction. Ultrasound in Obstetrics Gynecology sep 2016;48(3):349-356. ISSN 09607692.

[5] Doste R, Soto-Iglesias D, Bernardino G, Alcaine A, Sebastian R, Giffard-Roisin S, Sermesant M, Berruezo A, Sanchez-Quintana D, Camara O. A rule-based method to model myocardial fiber orientation in cardiac biventricular geometries with outflow tracts. International Journal for Numerical Methods in Biomedical Engineering apr 2019; 35(4). ISSN 20407947.

[6] Costabal FS, Hurtado DE, Kuhl E. Generating Purkinje networks in the human heart. Journal of biomechanics 2016; 49(12):2455-2465.

[7] Cardone-Noott L, Bueno-Orovio A, Minchole A, Zemzemi $\mathrm{N}$, Rodriguez B. Human ventricular activation sequence and the simulation of the electrocardiographic QRS complex and its variability in healthy and intraventricular block conditions. EP Europace 2016;18(suppl_4):iv4-iv15.

[8] Dux-Santoy L, Sebastian R, Rodriguez JF, Ferrero JM. Modeling the different sections of the cardiac conduction system to obtain realistic electrocardiograms. Proceedings of the Annual International Conference of the IEEE Engineering in Medicine and Biology Society EMBS 2013; 6846-6849. ISSN 1557170X.

[9] Mountris KA, Sanchez C, Pueyo E. A novel paradigm for in silico simulation of cardiac electrophysiology through the mixed collocation meshless petrov-galerkin method. In 2019 Computing in Cardiology (CinC). IEEE, 2019; 1-4.

[10] Mincholé A, Zacur E, Ariga R, Grau V, Rodriguez B. MRIbased computational torso/biventricular multiscale models to investigate the impact of anatomical variability on the ECG QRS complex. Frontiers in Physiology 2019;10:1103.

[11] Dower GE, Machado HB, Osborne J. On deriving the electrocardiogram from vectorcardiographic leads. Clinical Cardiology 1980;3(2):87-95. ISSN 0160-9289.

[12] Bukhari HA, Palmieri F, Ferreira D, Potse M, Ramírez J, Laguna P, Sánchez C, Pueyo E. Transmural ventricular heterogeneities play a major role in determining T-wave morphology at different extracellular potassium levels. In 2019 Computing in Cardiology (CinC). IEEE, 2019; 1-4.

Address for correspondence:

Freddy L Bueno-Palomeque GIIB-UPS, Universidad Politécnica Salesiana, Calle Vieja 12-30 and Elia Liut, 010102, Cuenca, Ecuador fbueno@ups.edu.ec 